\title{
Mitigação de Interferência entre Tecnologias Sem Fio em Ambientes Inteligentes
}

\author{
Francisco A. Rocha $^{1}$, Eduardo E. Mosca ${ }^{1}$, Gabriel G. Silva ${ }^{1}$, Rafael L. Gomes ${ }^{1}$ \\ ${ }^{1}$ Universidade Estadual do Ceará (UECE) \\ Fortaleza - CE - Brasil \\ \{alexandre.rocha, eduardo.mosca, gabriel.galdino, rafaellgom\}@larces.uece.br
}

\begin{abstract}
The Internet of Things (IoT) emerged as a paradigm for evolving daily tasks and implementing new services in environments such as homes, cities, campuses and so on. Transforming them into Intelligent Environments (or AIs). An AI is made up of heterogeneous wireless devices, some of which are mobile. These heterogeneous devices have a cross interference problem, since most of them use the $2.4 \mathrm{GHz}$ ISM band for communication. The cross-interference problem directly affects the quality of service $(Q o S)$, especially the low power transmission devices that provide the basis for services performed at the top of the network. Within this context, this article presents the Cross Interference Mitigation (MIC) mechanism, which aims to mitigate the problem of cross interference in intelligent environments. The MIC mechanism considers the mobility of the devices and their communication capacity to perform an appropriate wireless channel assignment. The results, from the experiments carried out, suggest that the MIC minimizes the interference of the applied devices, while significantly reducing the computational time, when compared to the existing approaches.
\end{abstract}

Resumo. A Internet das Coisas (IoT) surgiu como um paradigma para evoluir as tarefas diárias e implantar novos serviços em ambientes como casas, cidades, campus e etc. Transformando-os em Ambientes Inteligentes (ou AIs). Um AI é composto por dispositivos sem fio heterogêneos, sendo que parte deles são móveis. Esses dispositivos heterogêneos apresentam um problema de interferência cruzada, uma vez que a maioria deles usa a banda ISM de $2.4 \mathrm{GHz}$ para comunicação. O problema de interferência cruzada afeta diretamente a qualidade do serviço (QoS), principalmente os dispositivos de baixa potência de transmissão que fornecem a base dos serviços executados no topo da rede. Dentro desse contexto, este artigo apresenta o mecanismo Mitigação de Interferência Cruzada (MIC), o qual visa mitigar o problema de interferência cruzada em AIs. O mecanismo MIC considera a mobilidade dos dispositivos e sua capacidade de comunicação para realizar uma atribuição de canais sem fio adequada. Os resultados, a partir dos experimentos realizados, sugerem que o MIC minimiza a interferência dos dispositivos aplicados, enquanto reduz significativamente o tempo computacional, quando comparado às abordagens existentes.

\section{Introdução}

Hoje em dia a sociedade necessita de ambientes mais inteligentes, os quais disponibilizam serviços para o usuário final. Esse principio de Ambientes Inteligentes (AIs) vêm sendo 
implantado em várias áreas como: Cidades Inteligentes, Prédios Inteligentes, Casas Inteligentes, Indústria 4.0, E-Health entre outros. AIs são compostos por dispositivos de Internet das Coisas (IoT) (como sensores e atuadores) e dispositivos móveis (notebooks, smartphones, tablets, etc) [Jiang et al. 2019]. Essa heterogeneidade trás novos desafios relacionados ao gerenciamento, planejamento e comunicação dos AIs.

Os dispositivos dentro do AI se comunicam com a Internet ou com outros dispositivos através de uma Rede Local Sem Fio (Wireless Local Area Network - WLAN). Uma WLAN é composta de diversos Pontos de Acesso (Access Point - AP), os quais servem de ponto de comunicação. Tanto os dispositivos móveis quanto os dispositivos IoT utilizam a tecnologias sem fio para a comunicação. As tecnologias mais populares para esses AIs são Wifi e Zigbee, sendo que ambas tecnologias usam a frequência de comunicação 2.4 GHz ISM (Industrial, Scientific and Medical) [Ma et al. 2019]. O Wifi é o principal meio de comunicação dos dispositivos móveis dos usuários, enquanto o Zigbee é amplamente utilizado em dispositivos IoT devido à capacidade de transmissão e limites do uso de energia [Seyedolhosseini et al. 2017].

Outra característica importante dos AIs é a mobilidade. Nos AIs, a maioria dos dispositivos de IoT são fixos, uma vez que eles são sensores e atuadores usados para monitorar e executar ações no espaço físico. Por outro lado, os nós Wifi (dispositivos dos usuários) são móveis, como por exemplo smartphones, tablets, notebooks, etc. Em um determinado momento o nó Wifi está conectado a um certo AP, mas quando o usuário se move dentro do espaço físico vai migrando de um AP para outro e o canal do seu nó se ajustando ao AP correspondente. Assim, o nó Wifi pode interferir em dispositivos IoT que estejam próximos do novo AP.

Percebe-se então que a coexistência de aparelhos heterogêneos que usam a mesma frequência sem fio, mas que possuem diferentes características de transmissão (Antena, Potência, etc), é um problema muito complexo e ainda em aberto tanto para a indústria quanto para a comunidade científica. Faz-se necessário uma solução que consiga mitigar esta interferência cruzada, principalmente considerando as características de AIs, os quais possuem diversos APs cobrindo o espaço físico, enquanto realizam a intermediação da comunicação de centenas/milhares de dispositivos móveis e IoT num cenário inteligente como por exemplo, uma cidade inteligente ou campus inteligente.

Dentro desse contexto, este artigo apresenta o mecanismo Mitigação de Interferência Cruzada (MIC) para planejar a alocação dos canais dos dispositivos IoT e dos APs no contexto de AIs. O objetivo do mecanismo é reduzir o problema de interferência cruzada, considerando as seguintes questões: a mobilidade dos dispositivos móveis, a posição dos dispositivos IoT, o impacto da sobreposição dos canais (considerando a potência de transmissão e a atenuação de sinal).

O restante do artigo está organizado da seguinte forma. A Seção 2 detalha o trabalho existente relacionado à mitigação de interferência. A Seção 3 descreve o MIC e seus objetivos, enquanto a Seção 4 apresenta o resultado dos experimentos realizados. Por fim, a Seção 5 conclui o artigo.

\section{Trabalhos Relacionados}

Nesta seção, descrevemos os principais trabalhos relacionados a alocação de canais e minimização da interferência em redes sem fio. 
Wang et al. [Wang et al. 2019] propõe o DopplerFi, uma estrutura de comunicação que permite um canal de comunicação bidirecional entre Bluetooth Low Energy (BLE) e Wi-Fi por meio da injeção de deslocamentos Doppler artificiais, que podem ser decodificados por detecção os padrões no desmodulador Gaussian frequency shift keying (GFSK) e Channel State Information (CSI).

Jiang et al. [Jiang et al. 2019] explora a informação da densa camada física para comunicação de tecnologia cruzada, emulando a estrutura ZigBee com um rádio Bluetooth. A proposta alcança uma conformidade de padrão duplo e transparência por apenas modificar o payload dos quadros Bluetooth, não havendo a necessidade de modificar o remetente Bluetooth ou o receptor Zigbee. Contudo, a proposta não considera a interferência cruzada vinda de dispositivos Wifi presentes no AI.

Ponte et al. [da Ponte et al. 2019] apresenta um algoritmo, chamado CASH, para minimizar a interferência em casas inteligentes por meio da atribuição de canais sem fio adequados, reduzindo o problema de coexistência por meio de uma heurística. Os autores consideram uma casa inteligente composta de dispositivos com tecnologia sem fio como Wifi, BLE e Zigbee. Apesar da diminuição da interferência, o algoritmo apresentado possui um alto tempo de computação, impedindo seu lançamento no contexto dos AIs que exigem escalabilidade e têm grandes números de dispositivos IoT.

Seyedolhosseini et al. [Seyedolhosseini et al. 2017] apresenta a formulação para mitigar a interferência entre os dispositivos Wifi e Zigbee baseando-se na Taxa de Ruído de Interferência de Sinal (SINR), que mede a qualidade do sinal de transmissão. O autor utiliza o SINR junto com o algoritmo Kruskal para determinar o menor caminho entre os nós Zigbee de maneira que não causem perdas de pacotes nos nós Wifi. Todavia, a formulação proposta não considera a mobilidade dos nós, tal como os canais adequados a serem atribuídos aos dispositivos.

Chen et al. [Chen et al. 2019] propõe uma técnica para mitigar a interferência e, consequentemente, a colisão entre as tecnologias Wifi e Zigbee. A técnica proposta mescla o pacote 6LoWPAN para transmitir o pacote agregado posteriormente em um pacote Wifi, suspendendo a transmissão Zigbee com pacotes 6LoWPAN para evitar as colisões. Essa técnica possui dois problemas: a perda de um pacote resulta em uma perda de muita informação; e, a informação transmitida alcança o destinatário com um atraso considerável. Esses problemas podem comprometer a QoS dos serviços no topo do AI.

Para o nosso conhecimento, nenhum dos trabalhos encontrados na literatura, foca no problema da interferência cruzada das tecnologias sem fio Wifi e Zigbee, assim como a mitigação da interferência por dispositivos heterogêneos em AIs, considerando os problemas de mobilidade e comunicação. Portanto, este artigo apresenta um algoritmo para atribuir canais sem fio mais adequados as tecnologias Wifi e Zigbee, pois são tecnologias mais comuns em AIs.

\section{Proposta}

Nesta seção apresenta-se o mecanismo Mitigação de Interferência Cruzada (MIC), que visa realizar o planejamento na alocação de canais para as tecnologias Wifi e Zigbee mais adequado, com o intuito de reduzir a interferência sofrida principalmente pelas tecnologias com menor potência. Num AI, o algoritmo combina conjuntos de canais heterogêneos na busca das menores taxas de interferência, usando um modelo para estimar a 
interferência entre eles. Na Subseção 3.1 descreve-se como os dispositivos heterogêneos interagem entre si e como os fatores de interferência com base nos canais usados podem ser calculados. Na Subseção 3.2 apresenta-se o modelo de atenuação de sinal aplicado. Na Subseção 3.3 descreve-se o modelo de interferência entre dois dispositivos, considerando os aspectos de fator de interferência e atenuação de sinal. Por fim, a Subseção 3.4 detalha-se o mecanismo MIC proposto neste artigo.

\subsection{Fator de interferência entre canais sobrepostos}

O calculo da interferência entre os dispositivos é uma extensão do modelo proposto por Akl et al. [Akl and Arepally 2007], que calcula somente a interferência entre os dispositivos Wifi. Portanto, a fórmula da referência [Akl and Arepally 2007] é impraticável no contexto dos AIs, pois não considera dispositivos de menor potência que usam outras tecnologias para se comunicar e que são afetados pelo Wifi, como por exemplo o Zigbee.

No entanto, neste trabalho é apresentada uma evolução da fórmula existente proposta por [Akl and Arepally 2007], foram adicionados aspectos necessários para sua aplicação no contexto de AIs. Assim, a equação 2 proposta neste trabalho permite o cálculo da interferência entre dispositivos heterogêneos coexistentes em um mesmo ambiente. Foi adicionado também um fator de interferência entre dois canais heterogêneos sobrepostos (tabela completa disponível ${ }^{1}$ ). Com base nas análises descritas por Natarajan et al. [Natarajan et al. 2016]: Wifi causa interferência no Zigbee, mas apenas sofre interferência de outro dispositivo Wifi; e, Zigbee sofre interferência de dispositivos Wifi e isso causa interferência em dispositivos de menor potência e canal diminuto (como BLE).

Na prática o que ocorre entre dois dispositivos Wifi, é que a interferência é total (ou 100\%) se o mesmo canal for atribuído para um aparelho vizinho, e este valor é reduzido por um fator de 0.2 (ou 20\%) cada vez que você incrementa o canal. Por exemplo, se você designar o canal número 1 para um dispositivo Wifi, e seu vizinho usa o canal 2 , o fator de interferência é 0.8 ou $80 \%$. Assim, o fator de interferência vai reduzindo de acordo com a sobreposição. Então, quando há um espaçamento de cinco canais ou mais do Wifi, o fator de interferência é zero (ou $0 \%$ ), pois os canais não se sobrepõem (por exemplo, canais 1 e 6 ).

Por outro lado, o Zigbee não possui canais sobrepostos. Contudo, quando consideramos os aspectos do cenário heterogêneo (com dispositivos Wifi e Zigbee), tem-se que canais Wifi e Zigbee sobrepostos resultam em uma interferência total (ou 100\%) dos dispositivos Wifi sobre Zigbee. Por outro lado, a interferência dos dispositivos Zigbee sobre Wifi é zero (ou 0\%).

\subsection{Modelo de Atenuação de Sinal}

A atenuação de sinal é a perda da força de um sinal ao se propagar pelo ar, ou seja, a potência de transmissão. A distância entre dois dispositivos é usada para calcular a energia perdida (de acordo com a referência [Committee et al. 2003]) e mostrada na Equação 1. Assim, para uma frequência operacional de $2.4 \mathrm{GHz}$, a potência do sinal não é afetada quando a distância entre o emissor e o receptor é menor ou igual a 0,5 metros, resultando em uma atenuação de sinal (PL) igual a 1.

\footnotetext{
${ }^{1}$ https://github.com/rodrigoparente/interference-factor-w/
} 


$$
P L(d)=\left\{\begin{array}{l}
40.2+20 \cdot \log _{10}^{(d)}, 0.5 m<d, \leq 8 m \\
58.5+33 \cdot \log _{10}^{(d / 8)}, d>8 m
\end{array}\right.
$$

\subsection{Modelo de interferência}

O modelo de interferência calcula a interferência entre dois dispositivos heterogêneos, o qual é aplicado pelo mecanismo proposto (descrito na Subseção 3.4) para calcular a quantidade total de interferência que um dispositivo sofre. Durante o restante do artigo será usada a seguinte notação: $I_{i, j}^{z, m}$ é a interferência que o dispositivo $i$ usando o canal $z$ sofre de um dispositivo $j$ usando o canal $m ; P_{j}^{m}$ é a potência nominal $(\mathrm{dBm})$ que o dispositivo $j$ emite seu sinal sem fio pelo canal $m ; W^{z, m}$ é a quantidade de interferência que o canal $m$ causa ao canal $z$ (descrito na Seção 3.1); $P L(d)$ é a atenuação do sinal à distância $d$ para frequências operando a $2.4 \mathrm{GHz} ; A$ são APs Wifi; $B$ são APs Zigbee; $W$ são canais Wifi; $Z$ são Canais Zigbee; $h_{c}$ é o canal usado pelo dispositivo ou AP $h$; e, $\operatorname{Random}(L, M)$ é a função que escolhe aleatoriamente um canal da lista $M$ aos dispositivos da lista $L$.

A modelagem de interferência é descrita na Equação 2. A partir das informações sobre o fator de interferência e de atenuação do sinal pode-se determinar a interferência $I_{i, j}^{z, m}$ que o dispositivo $i$ usando o canal $z$ sofre de um dispositivo $j$ usando o canal $m$.

$$
I_{i, j}^{z, m}=\frac{W^{z, m} \times P_{j}^{m}}{P L(d)}
$$

\subsection{Mitigação de Interferência Cruzada (MIC)}

Esta subseção descreve o funcionamento do mecanismo proposto, que além de mitigar a interferência no ambiente, visa determinar a solução mais adequada em um curto espaço de tempo, visto que as cidades inteligentes são ambientes dinâmicos e com alto número de dispositivos móveis. A partir desses princípios, o mecanismo MIC é baseado em dois algoritmos: (I) O Algoritmo 1 percorre as possíveis combinações com interferência entre os APs Wifi e Zigbee, calculando a possível interferência entre os APs de tecnologias diferentes quando alocados certos canais, considerando a distância entre eles; e, (II) o Algoritmo 2, o qual é executado posteriormente dentro do Algoritmo 1, a fim de buscar o canal com a menor interferência na busca local do cenário em questão.

O Algoritmo 1, inicialmente, recebe uma lista de APs Wifi e Zigbee (definida como $A$ e $B$, respectivamente) como entrada, onde ambas tecnologias recebem canais aleatórios (linhas 1 e 2). Entre as linhas 3 e 12, o algoritmo inicia a estratégia gulosa para percorrer possíveis soluções de alocação de canais para os APs presentes (listas $A$ e $B$ ) e avaliar o impacto destas na interferência entre os dispositivos.

Na linha 5 dois APs são selecionados, então é verificado se são dos tipos WiFi e Zigbee respectivamente e se a interferência entre eles é maior que zero, pois em alguns casos devido a distância entre eles ou por já estarem com canais sem sobreposição não precisam ser modificados naquele momento, mas se a interferência existir, então na linha 6 é chamado o Algoritmo 2 que vai receber o AP Zigbee como parâmetro de entrada, buscar o canal com a menor interferência para lhe atribuir, com isso minimizar o nível de interferência encontrado no mesmo. 
Na linha 8 se a ordem dos APs selecionados for inversa(Zigbee e Wifi) em comparação com a linha 5, então é feito o mesmo processo de verificação dos APs selecionados. É verificado se há interferência, se houver é chamado o mesmo Algoritmo 2 e passado como parâmetro agora um AP Wifi selecionado, então o Algoritmo 2 retorna o canal que mais reduzir a interferência encontrada no mesmo.

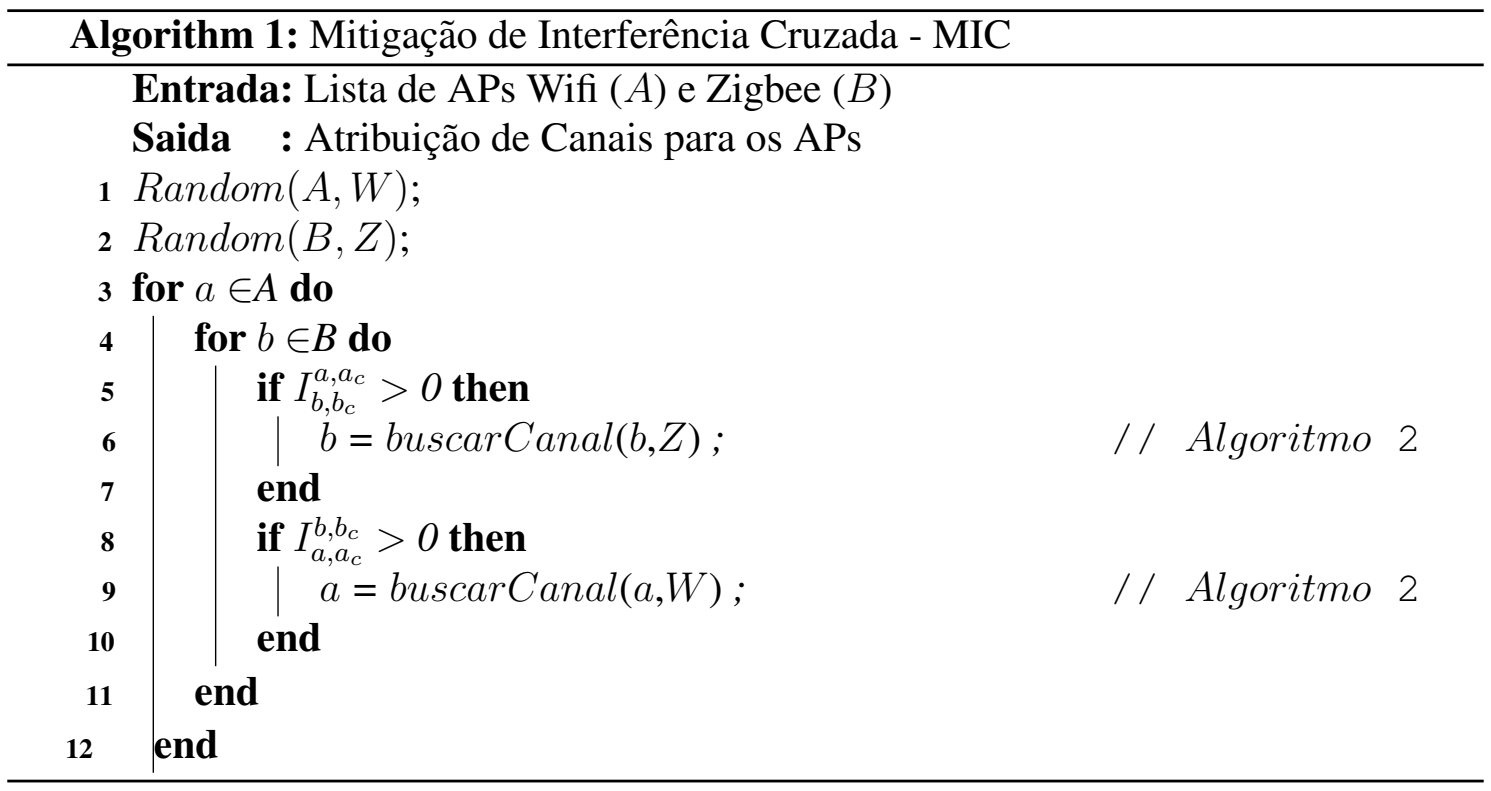

O Algoritmo 2 recebe como entrada um AP que pode ser Wifi ou Zigbee, e a lista de canais $L$ da tecnologia em questão. Na linha 1 a variável interfGeral recebe o valor de um teste da interferência de todos os APs naquele momento, a qual é calculada pela função teste. Na linha 2 a variável best é inicializada como vazia (sem canal até o momento). Na linha 3 é iniciado um laço que percorre a lista de canais do AP (Wifi ou Zigbee). Na linha 4 é feita a atribuição do canal $l$ testado no momento. Na linha 5 é calculada a interferência geral de todos os APs no momento (interf Atual), a qual é verificada se esta é menor que a inter fGeral. Se o resultado for positivo, é porque o canal recém atribuído conseguiu reduzir a interferência geral, então a variável interfGeral é atualizada para o valor da interfAtual e o best também recebe o canal que obteve a menor interferência (linhas 8 e 9, respectivamente). Este processo é repetido até se verificar todos os possíveis canais (13 no caso de Wifi e 16 no caso de Zigbee). Assim, ao final do laço, todos os canais do dispositivo terão sido testados e a variável best com o canal de menor interferência é retornado.

A proposta do MIC foca no tratamento da interferência tendo como base os APs (tanto Wifi quanto Zigbee), porque o número deles no AI é menor quando comparado ao de dispositivos dos cenários propostos, o que permite uma maior escalabilidade num cenário típico de um AI com diversos dispositivos heterogêneos com mobilidade. Ao analisar os APs, o mecanismo MIC tem uma visão geral da interferência de todos os dispositivos no cenário e propôr uma solução de mitigação de interferência num curto espaço de tempo, visto que os dispositivos conectados ao AP usam o mesmo canal definido neste.

Sobre o ponto de vista da escalabilidade, o MIC executa com uma complexidade de $O\left(N^{2} *(A * B)\right)$, onde $N$ é o número de dispositivos no ambiente, $A$ e $B$ são os 


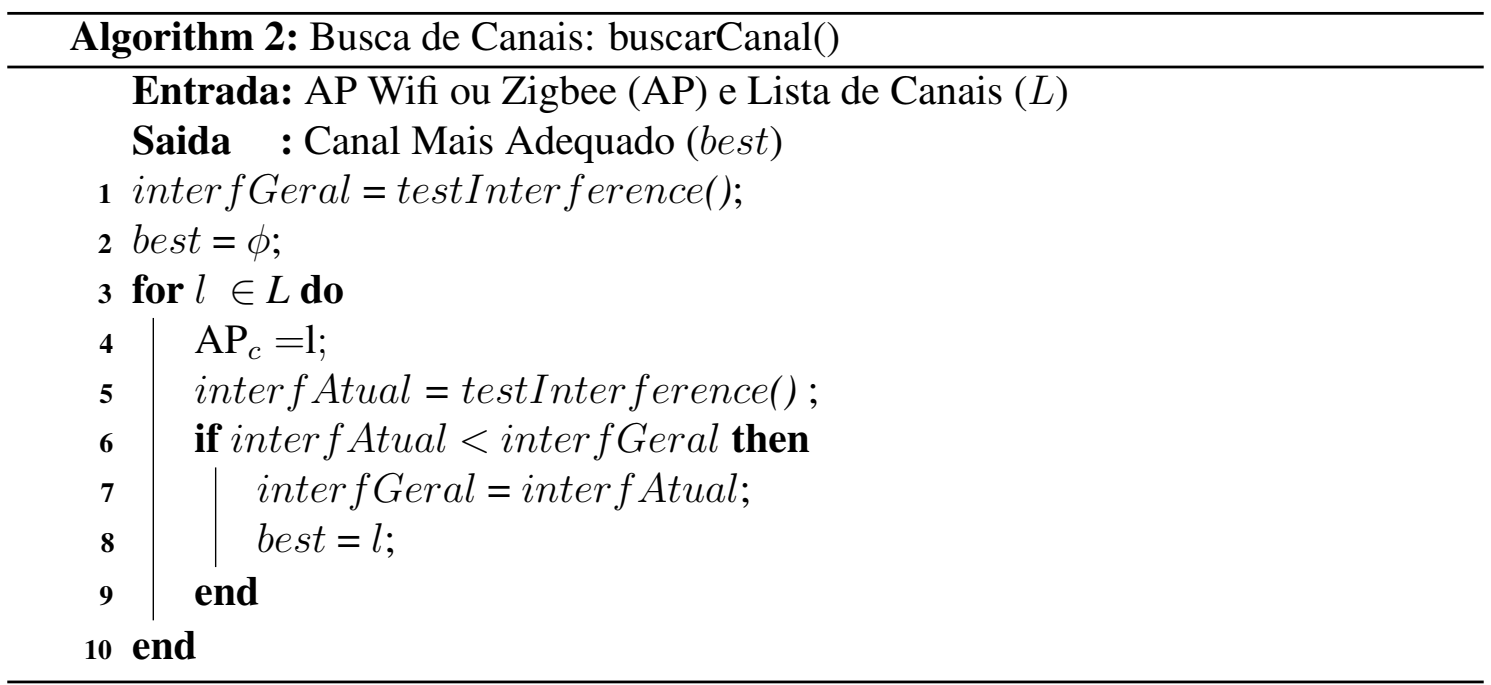

números de APs Wifi e Zigbee, respectivamente. Esta complexidade é resultante principalmente da necessidade de avaliar a interferência do AI no momento da análise. Quando comparado com as soluções existentes (analisadas na Seção 2), que possuem complexidades exponenciais em relação ao número de dispositivos no AI, tem-se uma solução viável a ser implantada no contexto de Cidades Inteligentes.

\section{Experimentos}

Esta seção apresenta os experimentos realizados para avaliar o mecanismo MIC. A Subseção 4.1 apresenta as configurações do cenário usado nos experimentos, enquanto que a Subseção 4.2 apresenta uma análise sobre os resultados.

\subsection{Configuração do Cenário}

Os experimentos foram realizados em um simulador desenvolvido para analisar os aspectos de rede sem fio de $\mathrm{AIs}^{2}$, o qual considera tanto as características de dispositivos heterogêneos e disposição física destes pelo ambiente.

Considerando um contexto de Cidades Inteligentes, foram definidos nove cenários contendo um número diferente de dispositivos sem fio e pontos de acesso: 10 APs com 200, 300 e 400 dispositivos; 15 APs com 300, 450 e 500 dispositivos; e, 20 APs com 400, 600 e 800 dispositivos. O tamanho do espaço físico definido foi de 400 por 400 metros. A posição dos dispositivos e dos pontos de acesso foram distribuídos aleatoriamente em cada simulação realizada levando em consideração a mobilidade aleatória dos mesmos. Cada dispositivo possui apenas uma tecnologia sem fio, onde 60\% deles usam Wifi, $40 \%$ dos dispositivos usam Zigbee. Esta estratégia visa tornar a avaliação dos resultados mais próxima do contexto de Cidades Inteligentes, onde há um número grande de dispositivos em relação ao número de APs, bem como a mobilidade dos dispositivos é imprevisível. Para cada cenário especificado foram executados 30 experimentos e os resultados são apresentados com um intervalo de confiança de $95 \%$.

Durante os experimentos, além do MIC, foram avaliadas três abordagens existentes: (a) O algoritmo Cash [da Ponte et al. 2019] proposto para minimizar interferência

\footnotetext{
${ }^{2}$ https://github.com/alexandresobral2004/projeto_mestrado
} 
em Casas Inteligentes, o qual considera a interferência de todos os dispositivos; (b) Atribuição do mesmo canal para todos os APs (chamada de Same), abordagem essa aplicada nos cenários com dispositivos espaçados e de alta mobilidade que realizam constantes migrações de AP; e, (c) Atribuição aleatória pra todos os APs (chamada de Random), que a partir de uma distribuição uniforme escolhe algum canal dos possíveis na tecnologia (13 canais pra Wifi e 16 pro Zigbee).

\subsection{Resultados}

Os experimentos avaliaram os seguintes aspectos: Interferência (Figuras 1(a), 1(b) e 1(c)), sendo a quantidade total de interferência experimentada pelos dispositivos no ambiente, levando em consideração a movimentação dos mesmos, Tempo de execução (Figuras 1(d), 1(e) e 1(f)), representando o tempo para encontrar uma solução e Entrega de pacotes 1(g).

Como pode ser observado nos resultados da Figura 1, o MIC obtém as menores taxas de interferência quando comparado com as abordagens existentes. O uso do Random apresenta-se interessante para um cenário com poucos dispositivos, no entanto quando a quantidade de dispositivos é grande, a interferência aumenta consideravelmente. O uso da abordagem Same segue o mesmo princípio, porém atinge taxas ainda maiores de interferência e também se torna impraticável no cenário proposto.

O uso do mecanismo MIC resultou numa redução significativa de interferência (em torno de 50\%) quando comparado ao Cash no cenário de 10 APs (Figura 1(a)). No cenário de 15 APs (Figura 1(b)) a redução de interferência do MIC se torna ainda mais significativa, reduzindo em torno de $60 \%$ a interferência comparado com o algoritmo Cash. Por fim, no cenário de 20 APs (Figura 1(c)) a redução da interferência chega a $70 \%$ do valor obtido pelo Cash.

O algoritmo Cash apresenta um aumento de interferência exponencial a medida que o número de APs e dispositivos cresce de acordo com o cenário. Este fato ocorre pois o Cash verifica a interferência de todos os dispositivos, mesmo que estejam fora do raio de alcance uns dos outros ou em canais não sobrepostos. Esta estratégia torna-se aplicável somente em casos com alta densidade de nós em um espaço físico diminuto (como por exemplo Casas Inteligente). Portanto, o tempo que o Cash leva para buscar o melhor cenário em relação a mitigação da interferência também é muito longo quando comparado ao MIC, conforme pode ser visualizado nas Figuras 1(d), 1(e) e 1(f).

Com relação ao tempo de execução do mecanismo MIC, este apresenta um desempenho adequado para o contexto de AIs, visto que espera-se que a definição dos canais alocados no ambiente possa ser reavaliada periodicamente. Esta necessidade vem da dinamicidade do ambiente, o qual possui mobilidade dos dispositivos, bem como precisa atender os requisitos de QoS esperado pelos serviços que executam no topo da infraestrutura de rede. Por fim, a figura $1(\mathrm{~g})$ mostra um aumento médio de $10 \%$ no número de pacotes entregues em relação ao Cash.

A partir dos resultados, torna-se inviável a aplicação do algoritmo Cash no âmbito de uma cidade inteligente ou de um ambiente inteligente onde o número de dispositivos é grande e a mobilidade é dinâmica. Assim, o mecanismo MIC apresenta os melhores resultados para aplicação num ambiente dinâmico e escalável, pois encontra um cenário com menor interferência dentro de um tempo adequado, bem como mitiga a interferência dos 


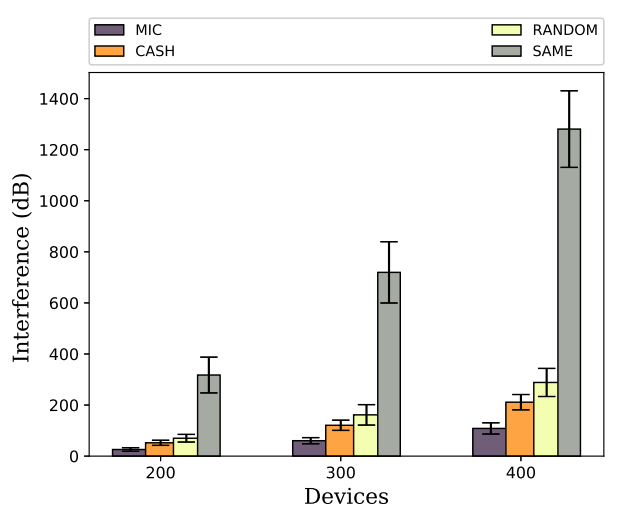

(a) Interferência entre 10 APs

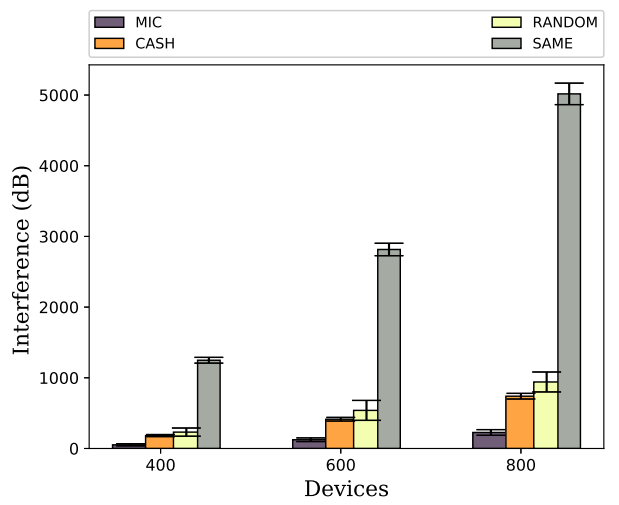

(c) Interferência entre 20 APs

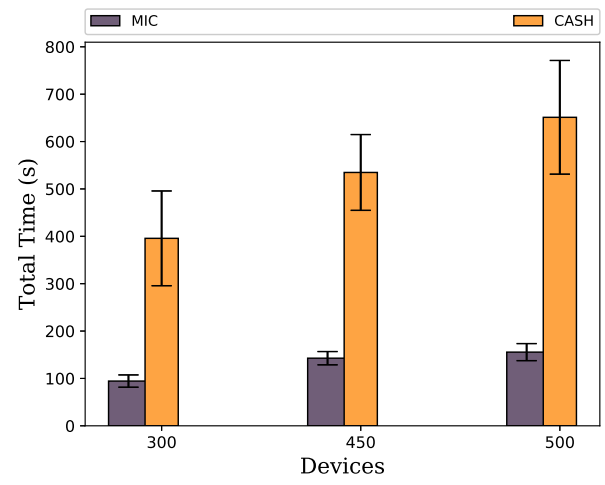

(e) Tempo com 15 APs

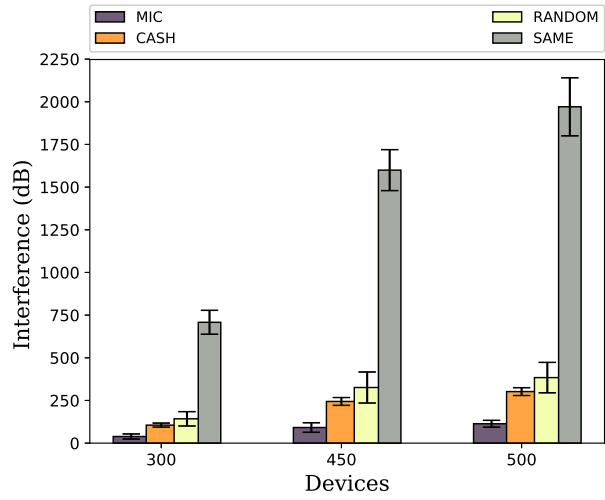

(b) Interferência entre 15 APs

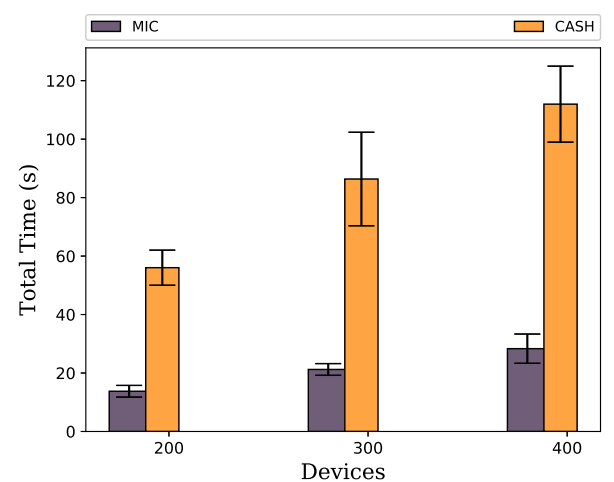

(d) Tempo com 10 APs

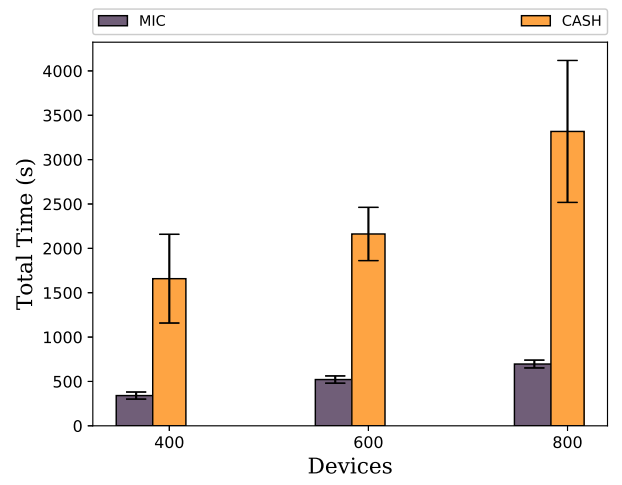

(f) Tempo com 20 APs

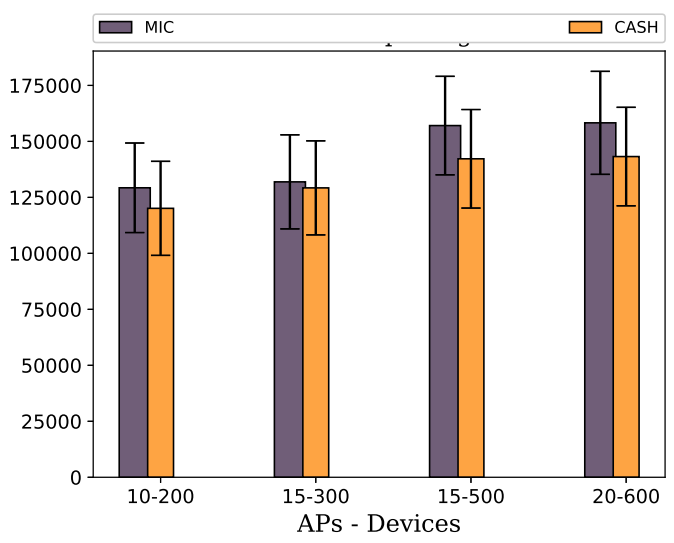

(g) Pacotes Entregues

Figura 1. Resultados 
dispositivos que estão dentro do mesmo raio de alcance e/ou em canais sobrepostos, com isso se torna mais rápido e consegue propor um cenário mais favorável aos dispositivos.

\section{Conclusão}

Atualmente, a frequência de $2.4 \mathrm{GHz}$ é a mais utilizada por diversos dispositivos heterogêneos em AIs, resultando em altas taxas de interferência, principalmente se os dispositivos forem dinâmicos. A fim de mitigar esse problema, este trabalho propôs o mecanismo MIC para minimizar a interferência cruzada entre os dispositivos heterogêneos levando em consideração a mobilidade dos mesmos e os aspectos de escalabilidade dos AIs. A partir dos experimentos realizados, o MIC mostrou-se mais eficiente que as abordagens existentes no que se refere a redução da interferência entre os dispositivos heterogêneos, redução do tempo gasto para encontrar o planejamento dos canais e aumentou a entrega de pacotes. Como trabalhos futuros, pretende-se avaliar os aspectos de Qualidade de Experiência (QoE) dos serviços quando o mecanismo proposto é utilizado.

\section{Referências}

Akl, R. and Arepally, A. (2007). Dynamic channel assignment in ieee 802.11 networks. In Portable Information Devices, 2007. PORTABLE07. IEEE International Conference on, pages 1-5. IEEE.

Chen, D., Khan, J., Javed, M. A., and Brown, J. (2019). Interference mitigation techniques for a dense heterogeneous area network in machine-to-machine communications.

Committee, L. M. S. et al. (2003). Coexistence of wireless personal area networks with other wireless devices operating in unlicensed frequency bands. IEEE Computer Society.

da Ponte, F. R. P., Gomes, R. L., Celestino, J., Pereira, W. F., and da Fonseca, N. L. S. (2019). Cash: A channel assigner algorithm for heterogeneous devices in smart homes. In 2019 IFIP/IEEE Symposium on Integrated Network and Service Management (IM), pages 624-628.

Jiang, W., Yin, Z., Liu, R., Li, Z., Kim, S. M., and He, T. (2019). Boosting the bitrate of cross-technology communication on commodity iot devices. IEEE/ACM Transactions on Networking, 27(3):1069-1083.

Ma, Z., Xiao, M., Xiao, Y., Pang, Z., Poor, H. V., and Vucetic, B. (2019). High-reliability and low-latency wireless communication for internet of things: Challenges, fundamentals, and enabling technologies. IEEE Internet of Things Journal, 6(5):7946-7970.

Natarajan, R., Zand, P., and Nabi, M. (2016). Analysis of coexistence between ieee 802.15. 4, ble and ieee 802.11 in the $2.4 \mathrm{ghz}$ ism band. In Industrial Electronics Society, IECON 2016-42nd Annual Conference of the IEEE, pages 6025-6032. IEEE.

Seyedolhosseini, A., Masoumi, N., and Modarressi, M. (2017). Performance improvement of zigbee networks in coexistence of wi-fi signals. In Proceedings of the 7th International Conference on Information Communication and Management, ICICM 2017, page 45-49, New York, NY, USA. Association for Computing Machinery.

Wang, W., He, S., Sun, L., Jiang, T., and Zhang, Q. (2019). Cross-technology communications for heterogeneous iot devices through artificial doppler shifts. IEEE Transactions on Wireless Communications, 18(2):796-806. 